\title{
Nonlinear Stress and Deformation Behaviour of Composite Sandwich Beams
}

\author{
E.E. Gdoutos ${ }^{1, a}$, I.M. Daniel ${ }^{2, b}$ \\ ${ }^{1}$ School of Engineering, Democritus University of Thrace, GR-671 00 Xanthi, Greece \\ ${ }^{2}$ Robert McCormick School of Engineering and Applied Sciences, Northwestern University, \\ Evanston, Illinois 60208, USA \\ a egdoutos@civil.duth.gr, ${ }^{b}$ imdaniel@northwestern.edu
}

Keywords: Nonlinearities, sandwich beams, foam cores, deformation, failure.

\begin{abstract}
The nonlinear load-displacement and normal stress distribution in composite sandwich beams made of unidirectional carbon/epoxy facings and PVC foam cores under bending was studied. The carbon/epoxy after an initial linear response exhibits a stiffening nonlinearity in tension and a softening nonlinearity in compression with the longitudinal strength in tension higher than that in compression. The foam core also presents a nonlinear stress-strain response. It was obtained that the load-displacement behaviour of the beam, after an initial linear part, is not linear. This behavior was modeled by an incremental strength of materials nonlinear analysis. The theoretical predictions were in good agreement with the experimental results. Furthermore, it was obtained that the neutral axis of sandwich beams under bending does not pass through the centroid of the cross section, but is displaced toward the tensile side of the beam. Experimental results by moire measurements of the in-plane horizontal displacements of the core material corroborated the analytical predictions. These findings imply higher compressive and smaller tensile stresses in the core, than those predicted for facings with identical stress-strain behaviour in tension and compression, and should be taking into consideration in the failure analysis of sandwich beams.
\end{abstract}

\section{Introduction}

Sandwich structures consisting of strong and stiff facings and light weight cores offer improved stiffness and strength to weight ratios compared to monolithic materials. Under flexural loading the facings carry almost all of the bending, while the core takes the shear loading and helps to stabilize the facings. Facing materials include metals and fiber reinforced composites. The latter are being used in advanced applications due to the large strength-to-weight ratio. The core materials mainly include honeycombs, foams and wood. Foam cores are widely used in sandwich construction. They include a large selection of foamed plastic materials with a variety of densities and shear moduli.

An extensive amount of work has been performed at Northwestern University on the mechanical behaviour and failure of sandwich beams under four- and three-point bending and cantilever beams made of unidirectional carbon/epoxy (AS4/3501-6) facings and a PVC closed-cell foam (Divinycell) cores [1-7]. The load-deflection behaviour of these materials consists of an initial linear portion followed by a nonlinear part. The deflection of the beam is the sum of the deflection due to bending of the facings and the shear of the core. The shear component of the deflection is not negligible as in monolithic materials, but of the same order of magnitude as the deflection due to bending and should be accounted for in the total deflection of the beam.

In the present work the effect of stress-strain behavior of facing and core materials on the nonlinear load-displacement response and stress distribution under bending of composite sandwich beams made of unidirectional carbon/epoxy facings and PVC foam cores was studied. Experiments show that the load-deflection curves consist of an initial linear part followed by a nonlinear portion. Also results by moiré measurements show that the neutral axis of sandwich beams under bending does not pass through the centroid of the cross section, but is displaced toward the tensile side of the 
beam. An analysis based on strength of materials was developed to predict the experimental observation.

\section{Theoretical considerations}

Consider a sandwich beam with rectangular cross section and facing and core materials that display a linear elastic behavior, subjected to a bending moment, $\mathrm{M}$, and shear force, $\mathrm{V}$. The flexural rigidity of the beam, $\mathrm{D}$, and the in-plane maximum normal stress, $\sigma$, and shear stress, $\tau$, in the core, for a low stiffness core and thin facings, are given by [1]

$$
\begin{aligned}
D & =\frac{b h_{f} d^{2}}{2 E} . \\
\sigma & =\frac{P L}{C_{1} b d^{2}}\left(\frac{E_{c}}{E_{f}}\right) \frac{h_{c}}{h_{f}} \\
\tau & =\frac{P}{C_{2} b h_{c}}
\end{aligned}
$$

where

$$
\mathrm{M}=\frac{\mathrm{PL}}{\mathrm{C}_{1}}, \quad \mathrm{~V}=\frac{\mathrm{P}}{\mathrm{C}_{2}}
$$

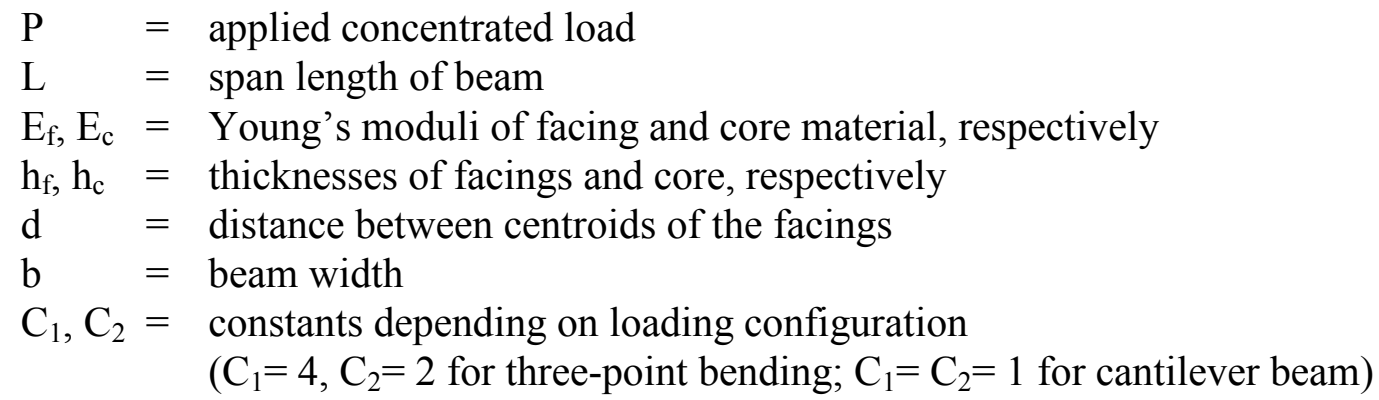

The maximum normal stress, $\sigma$, for a beam under three-point bending occurs under the load, while for a cantilever beam under end loading it occurs at the support. The shear stress, $\tau$, is constant along the beam span and through the core thickness, as verified experimentally [2].

The deflection of the beam is given by

$$
\delta_{p}=\frac{\mathrm{PL}^{3}}{24 \mathrm{Ebh}_{\mathrm{f}} \mathrm{d}^{2}}+\frac{\mathrm{PLh}_{\mathrm{c}}}{4 \mathrm{G}_{\mathrm{c}} \mathrm{bd}} .
$$

where the first term is the deflection due to bending and the second term is the deflection due to shear.

\section{Nonlinearities in the deformation of sandwich beams}

The above analysis is valid for linear elastic behavior of both core and facing materials and for small beam deflections. For nonlinear elastic behavior of the core and facings materials Eq. (5) can be used to determine the differential displacements corresponding to a load increment. In this case $\mathrm{E}$ 
and $\mathrm{G}_{\mathrm{c}}$ represent the tangent elastic modulus of the facing material and the shear modulus of the core.

For nonlinear elastic material behavior, an incremental nonlinear analysis is introduced. The critical value $\mathrm{P}_{\mathrm{i}}$ of the applied load $\mathrm{P}$ for which the linear elastic limit of the facings or core material is first exceeded is determined. The load is then increased by increments. The mean facings normal tensile or compressive stress corresponding to a bending moment $\mathrm{M}$ created by the applied load $\mathrm{P}$ is given by

$$
\sigma_{\mathrm{t}}=-\sigma_{\mathrm{c}}=\frac{\mathrm{M}}{\mathrm{bdh}}
$$

while the mean core shear stress is calculated from Eq. (3).

From the values of $\sigma_{\mathrm{t}}, \sigma_{\mathrm{c}}$ and $\tau$ and using the uniaxial stress-strain curves of the facings materials in tension and compression and the shear stress-strain curve of the core material the values of the tangent moduli are determined. Equation (5) is then used to calculate the deflection increment corresponding to a load increment $\Delta \mathrm{P}$. The total deflection of the beam at a load $\mathrm{P}$ is then given by

$$
\delta=\delta_{i}+\Sigma \Delta \delta
$$

where $\delta_{\mathrm{i}}$ is the deflection corresponding to the lowest value of the applied load when the proportional limit of any one of the materials is reached.

\section{Nonlinearities in the stress field of the sandwich beam}

Equations (2) and (3) apply to the case when both facings and core materials display the same linear elastic stress-strain behavior in tension and compression. When the stress-strain curve is not linear and/or the same in tension and compression, the above equations are not valid. This is the case of sandwich beams with carbon/epoxy facings and Divinycell core used in this study. These materials exhibit nonlinear stress-strain behavior, which is different in tension and compression. For this case, and under the assumption of linear strain distribution through the beam cross section, the distance of the neutral axis, $\mathrm{x}$, of the beam from the midpoint of the tensile side is given by:

$$
\mathrm{X}=\frac{\mathrm{h}}{1+\frac{\varepsilon_{\mathrm{c}}}{\varepsilon_{\mathrm{t}}}}
$$

where $\varepsilon_{\mathrm{c}}$, and $\varepsilon_{\mathrm{t}}$ are the compressive and tensile strains at the midpoints of the facings, and $\mathrm{h}$ is the height of the beam. For linear elastic behavior and identical stress-strain curves in tension and compression for the facing material, we obtain from Eq. (8) and for $\varepsilon_{\mathrm{t}}=\varepsilon_{\mathrm{c}}, \mathrm{x}=\mathrm{h} / 2$, that is, the neutral axis passes through the center of the cross-section of the beam $(x=h / 2)$.

\section{Experimental procedure and results Materials and specimens}

The sandwich beam facings were 8-ply unidirectional carbon/epoxy plates (AS4/3501-6), of 1mm thickness fabricated separately by autoclave molding. Uniaxial tensile and compressive tests were conducted primarily in the longitudinal direction in order to obtain the relevant constitutive behavior of the facing material. The compressive tests were performed using a fixture developed at Northwestern University [8]. The concept of the fixture is to transmit the initial part of the load through the tabs by shear loading and thereafter engage the ends to apply the additional load to 
failure by end loading. The longitudinal tensile and compressive stress-strain behavior for the AS4/3501-6 carbon/epoxy is shown in Fig. 1, where it is seen that the material exhibits a characteristic stiffening nonlinearity in tension and softening nonlinearity in compression.

Three core materials were investigated. One of them was aluminum honeycomb (PAMG 8.13/16 001-P-5052, Plascore Co.). The other core materials were two types of PVC closed-cell foam, Divinycell $\mathrm{H} 100$ and $\mathrm{H} 250$, with densities of 100 and $250 \mathrm{~kg} / \mathrm{m}^{3}$, respectively. The aluminum honeycomb material is highly anisotropic with much higher stiffness and strength in the throughthe-thickness direction (cell direction) than in the in-plane directions. For the characterization of the core material properties refer to $[9,10]$.

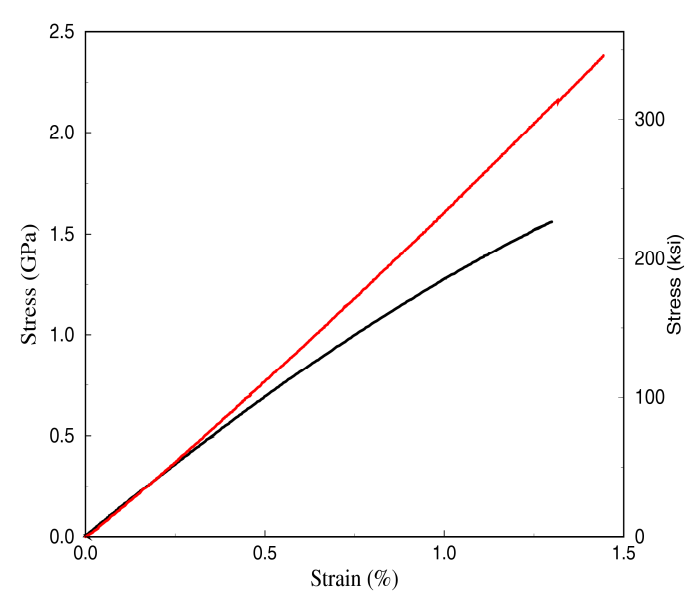

Figure 1: Stress-strain curves in tension (exhibiting hardening nonlinearity) and compression (exhibiting softening nonlinearity) of carbon/epoxy facings (AS4/3501-6)

\section{Testing of sandwich beams}

The honeycomb core was $2.54 \mathrm{~cm}$ wide and was machined from a $2.54 \mathrm{~cm}$ thick sheet along the stiffer in-plane direction. The $2.54 \mathrm{~cm}$ wide composite facings were machined from unidirectional plates, and were bonded to the top and bottom faces of the honeycomb core with FM73 M film adhesive. The assembly was cured under pressure in an oven following the recommended curing cycle for the adhesive. Sandwich beams were also prepared by bonding composite facings to foam cores of $2.54 \times 2.54 \mathrm{~cm}$ cross section using an epoxy adhesive (Hysol EA 9430). The adhesive was cured at room temperature by subjecting the sandwich beam to vacuum. The cured adhesive layer was $0.13 \mathrm{~mm}$ thick.

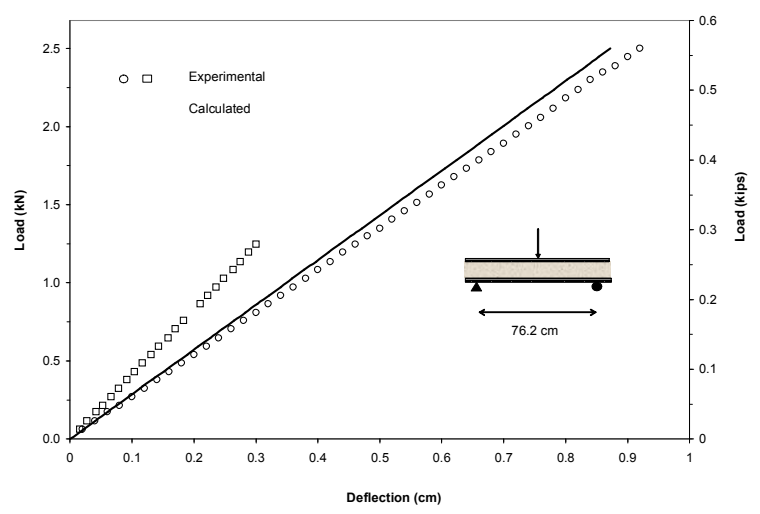

Fig. 2 Midspan (left) and quarter-span (right) load-deflection curves of a sandwich beam of length $\mathrm{L}=76.2 \mathrm{~cm}$ under three-point bending (circles denote experimental measurements, continuous lines denote theoretical predictions). 
Special fixtures were fabricated for beams subjected to three-point and four-point bending and for end-loaded cantilever beams. In studying the effects of pure bending special reinforcement was provided for the core at the outer sections of the beam to prevent premature core failures. Also, under three-point bending, the facings directly under concentrated loads were reinforced with additional layers of carbon/epoxy to suppress and prevent indentation failure. Only in the case when the indentation failure mode was studied there was no face reinforcement.

A series of three-point bend specimens and cantilevers with different span lengths were conducted. The specimens were loaded up to failure. The load-deflection curves were plotted for all tests. Figure 2 shows the load midspan and the load quarter span deflection curves for a beam of span $\mathrm{L}=76.2 \mathrm{~cm}$. A characteristic feature of the curve is that there is some small departure from linearity at an applied load $\mathrm{P}=2130 \mathrm{~N}$. This load corresponds to 84 percent of the maximum load. The load-deflection behavior of a beam of length $\mathrm{L}=25.4 \mathrm{~cm}$ is shown in Figure 3. Note that in this case there is a strong departure from linearity after a value of $\mathrm{P}=3180 \mathrm{~N}$. This value of $\mathrm{P}$ corresponds to 60 percent of the maximum load. The shear stress in the core for this load is 2.41 $\mathrm{MPa}$. This indicates that the nonlinearity in the load-deflection curve corresponds to the nonlinear behavior of the shear stress-strain curve of the core material that affects the shear component of the deflection of the beam. By comparing the nonlinear region for the two beams, we observe that it is larger for the shorter than for the longer beam. This is because of the higher effect of shear on the deflection of the shorter than the longer beam.

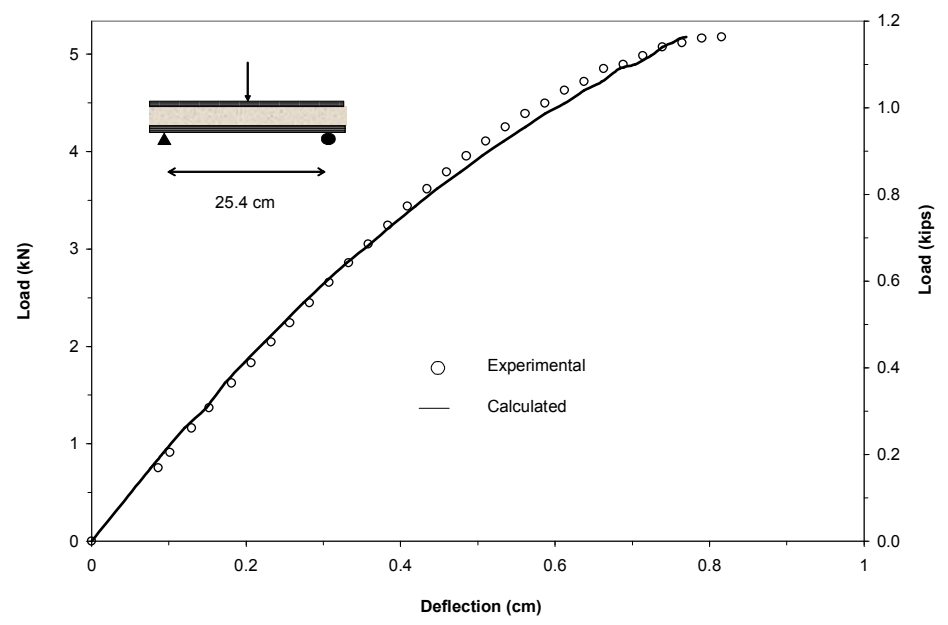

Fig. 3 Midspan load-deflection curves of a sandwich beam of length $\mathrm{L}=76.2 \mathrm{~cm}$ under three-point bending (circles denote experimental measurements, continuous lines denote theoretical predictions).

The deformation and failure mechanisms in the core were studied experimentally by means of moiré gratings. Figure 4 shows moiré fringe patterns for the horizontal, $u$, displacements in the core of a sandwich beam with aluminum honeycomb core under four-point bending. They were obtained with specimen gratings of 11.8 lines $/ \mathrm{mm}$ and a master grating of the same pitch with lines parallel to the vertical direction. The moiré fringe patterns of Fig. 4 corresponding to the horizontal $(u)$ displacements away from the applied load consist of nearly parallel and equidistant fringes from which it follows that the neutral axis of the beam does not pass though the centroid of the beam cross section but is displaced toward the tensile axis of the beam. In the following section a prediction of the position of the neutral axis of the beam will be made. 


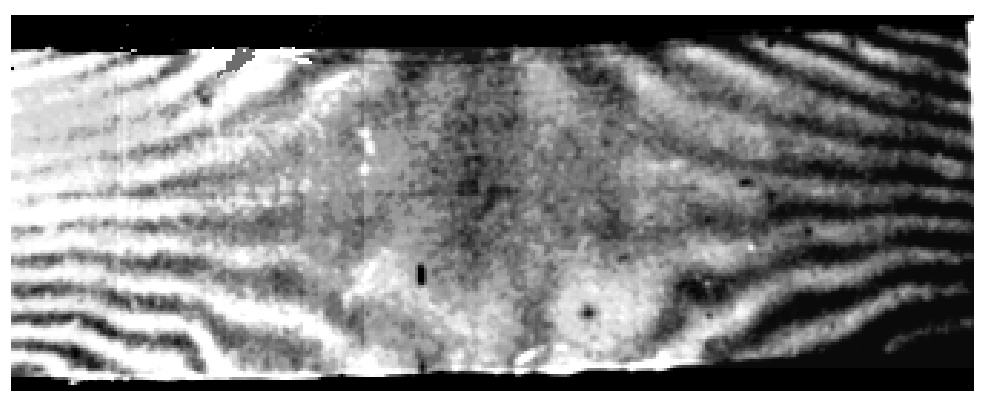

Figure 4: Moiré fringe pattern for the horizontal displacements in the core of a sandwich beam with honeycomb core under four-point bending.

\section{Prediction of location of neutral axis}

The neutral axis of the composite sandwich beam passes through the centroid of the beam cross section when the facing material exhibits the same linear elastic stress-strain behavior in tension and compression. This is not the case for the unidirectional carbon /epoxy facings used in this study. As can be observed in Figure 1, the stress-strain curves of the material in tension and compression show an identical initial linear portion up to a strain of 0.3 percent, followed by nonlinear behavior. The compression stress-strain curve is characterized by a softening nonlinearity, while the tensile stress-strain curve shows a hardening nonlinearity (Figure 1). This behavior of the facing material is responsible for the translation of the central axis of the sandwich beam from the midpoint of the cross section towards the tensile side. The location of the neutral axis can be predicted as follows:

Because of the high elastic modulus of the facings compared with the core material, it can be assumed that the applied moment is taken up entirely by the facings. The tensile, $\sigma_{t}$, and compressive, $\sigma_{\mathrm{c}}$, stresses in the beam are given by Eq. (6).

For a given applied moment, $\mathrm{M}$, the stresses $\sigma_{\mathrm{t}}$ and $\sigma_{\mathrm{c}}$ are first calculated from Eq. (6). From the values of these stresses and using the stress-strain curves of the facing material in tension and compression (Figure 1) the corresponding compressive, $\varepsilon_{\mathrm{c}}$, and tensile, $\varepsilon_{\mathrm{t}}$, strains are determined. Then, Eq. (8) is used to calculate the distance $\mathrm{x}$ of the neutral axis from the tensile side of the beam. From Figure 1 it is observed that for the same stress, the compressive strain is higher than the tensile strain $\left(\left|\varepsilon_{\mathrm{c}}\right|>\varepsilon_{\mathrm{t}}\right)$. Then, Eq. (3) implies that $\mathrm{x}<0.5 \mathrm{~h}_{\mathrm{c}}$, which indicates that the neutral axis is closer to the tensile side of the beam.

Figure 5 represents the variation of the normalized location of the neutral axis, $\mathrm{x} / \mathrm{h}$, versus the applied moment, M. Note that $\mathrm{x}=0.5 \mathrm{~h}_{\mathrm{c}}$ for an applied moment smaller than $280 \mathrm{Nm}$, which corresponds to a stress value equal to $0.434 \mathrm{GPa}$ at which the stress-strain curves of the carbon/epoxy material of the facings in tension and compression become nonlinear (Fig. 1). As the applied moment increases, the value of $\mathrm{x} / \mathrm{h}$ decreases, which means that the neutral axis of the beam recedes from the mid-point of the cross section and moves towards the tensile side of the beam. For a critical failure moment of $\mathrm{M}=1,000 \mathrm{Nm}$, which corresponds to the compressive strength of the carbon/epoxy facing of $\sigma_{\mathrm{c}}=1.55 \mathrm{GPa}$, the distance of the neutral axis from the tensile side of the beam is $\mathrm{x}=0.425 \mathrm{~h}$. 


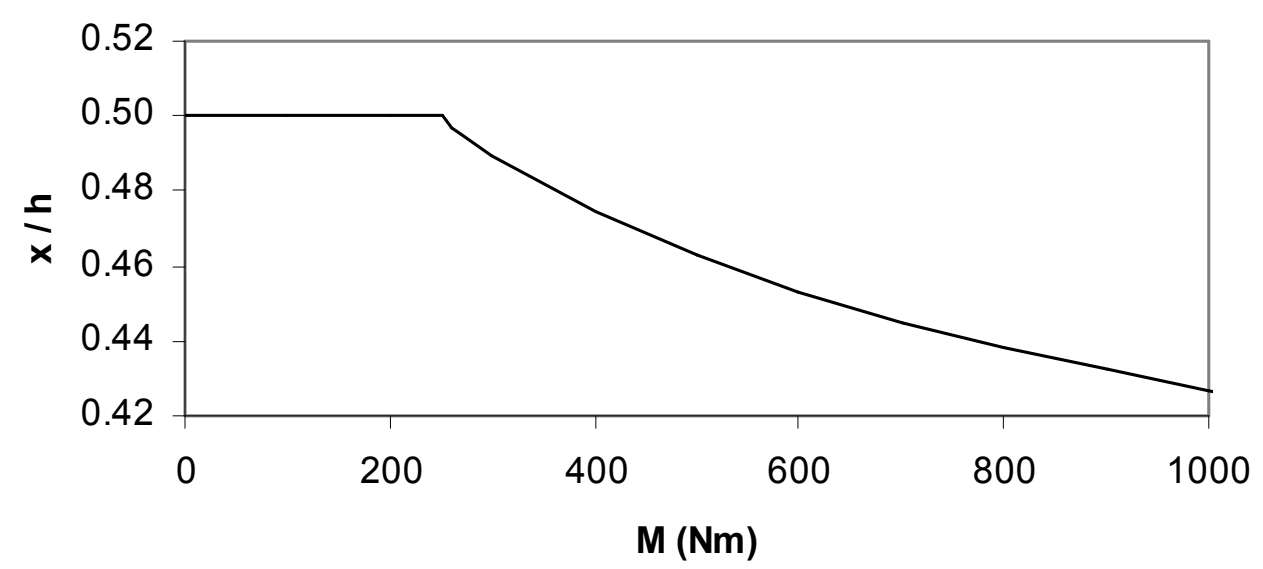

Figure 5: Normalized distance of the neutral axis from the tensile side of a beam versus applied moment.

Figure 6 represents the variation of the location of the neutral axis from the tensile side of a beam in three-point bending under a critical applied load which corresponds to failure of the compression facing of the beam. Note that the neutral axis passes through the centroid of the beam cross section near its ends, where the applied moment is small and the facing material is in the linear elastic range for tension and compression. As we move away from the ends of the beam and the applied moment increases, the neutral axis is shifted toward the tensile side of the beam. The maximum deviation of the neutral axis takes place under the load and is $\mathrm{x}=0.425 \mathrm{~h}$.

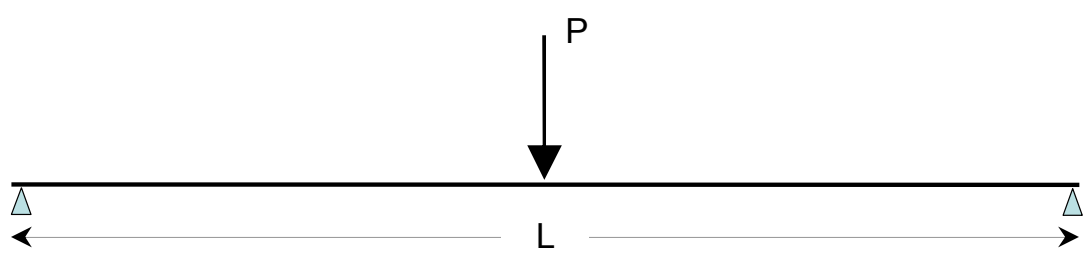

(a)

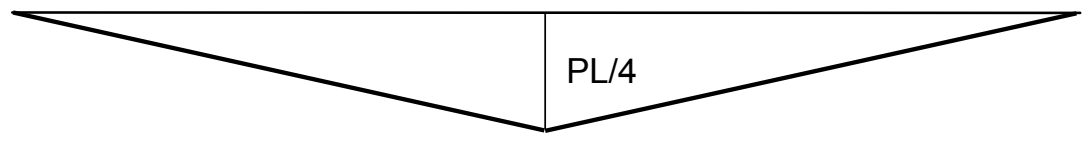

(b)

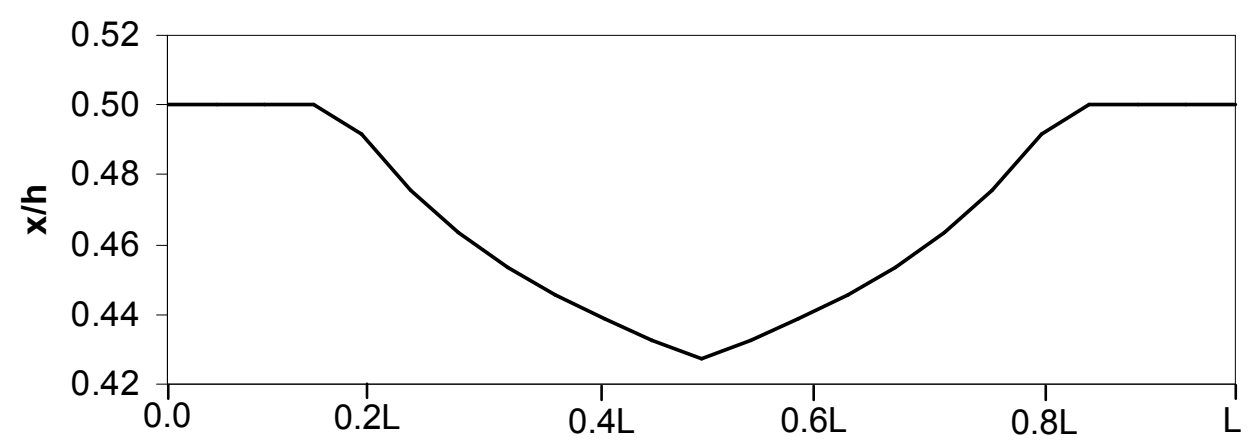

(c)

Figure 6: (a) Beam under three-point bending (b) Bending moment diagram, and (c) normalized distance of the neutral axis from the tensile side 


\section{Conclusions}

An investigation of the effect of the nonlinear stress-strain behavior of carbon/epoxy facings of composite sandwich beams on the location of the neutral axis of the beam was undertaken. The observed nonlinearity in the load-deflection curves was attributed to the nonlinear stress-strain behaviour of the facing and core materials. A mechanics of materials analysis was developed to predict the experimental load-deflection curves.

The neutral axis of the beam in the region of nonlinear deformation of the facings does not pass through the centroid of the beam cross section, but is displaced toward the tensile facing of the beam. A method for prediction of the location of the neutral axis was developed. The results of the present study suggest that higher compressive and lower tensile stresses than those calculated by classical bending theory are developed in the core material of the beam. It is established that the normal stresses in the core along the axis of the beam do not vary linearly and should be calculated by taking into account the nonlinear behavior of the facing and core materials in tension and compression.

\section{Acknowledgements}

This research was sponsored by the Office of Naval Research (ONR). We are grateful to Dr. Y.D.S. Rajapakse of ONR for his encouragement and cooperation. The authors wish to thank Eleftherios E. Gdoutos, undergraduate student in the Departments of Mechanical Engineering and Computer Science of Northwestern University, for his help in the calculations of the present work.

\section{References}

[1] Zenkert, D., An Introduction to Sandwich Construction, Chameleon, London, (1995).

[2] I.M. Daniel, E.E. Gdoutos, K.-A. Wang and J.L. Abot: 2001, Int. J. Dam. Mech. Vol. 11, (2002), p. 309

[3] E.E. Gdoutos, I.M. Daniel, and K.-A. Wang: Exp. Mech. Vol. 42, (2002), p. 426

[4] I.M. Daniel, E.E. Gdoutos and K.-A. Wang: Advan. Comp. Letters Vol. 11, (2002), p. 49

[6] J.L. Abot, I.M. Daniel, and E.E. Gdoutos: J. Sand. Struct. Mat. Vol. 4, (2002), p. 157

[7] E.E. Gdoutos, I.M. Daniel, K.-A. Wang: Mechanics of Materials Vol. 35, (2003), p. 511

[8] H.M. Hsiao, I.M. Daniel, and S.C. Wooh, J. Comp. Mat. Vol. 29, (1995), p. 1789

[9] E.E. Gdoutos, I.M. Daniel, and K.-A. Wang: Composites, Part A Vol. 33, (2002), p. 163

[10] I.M. Daniel, and J.L. Abot: Comp. Sci. and Techn. Vol. 60, (2000), p.2455 